Aus der chirurgischen Klinik zu Leipzig (Direktor der Klinik: Geh. Rat Prof. Dr. Trendelenburg, jetzt Geh. Med.-Rat Prof. Dr. Payr).

\title{
Zur Heilung der angeborenen Harnblasen- und Harnröhrenspalte.
}

Von Dr. W. Dünkeloh, Assistent der Klinik.

„Die Heilung der angeborenen Harnröhren- und Blasenspalte ist eines der schwierigsten und interessantesten Kapitel der plastischen Chirurgie und gewährt im Falle des Gelingens reiche Befriedigung." Dies sind die einleitenden Worte Trendelenburgs in seinem Vortrage, den er im Jahre I906 auf dem Chirurgenkongre $B$ in Amerika über die chirurgische Behandlung der Epispadie und Blasenspalte hielt. Trendelenburg konnte mit berechtigtem Stolz auf eine Reihe von Erfolgen zurückblicken, die er durch seine Methode in der Heilung der Blasenspalte erzielt hatte. Trotzdem sind immer wieder Bedenken laut geworden, die sich gegen diese Methode und vor allem gegen die Durchtrennung der Synchondrosen richten. Diese Bedenken durch Veröffentlichung von weiteren von Trendele $\mathrm{nburg}$ nach seiner Methode mit Erfolg operierten Fällen zu zerstreuen, ist der Zweck dieser Arbeit.

Bevor ich die Krankengeschichten bringe, möchte ich einen kurzen Überblick über die verschiedenen Operationsmethoden geben. Von den vielen operativen Versuchen haben sehr viele zurzeit nur mehr historisches Interesse, und es haben sich in der Neuzeit nur Methoden erhalten, die sich in folgende drei Gruppen einteilen lassen:

I. in die plastischen Methoden, die entweder mit Hautlappen aus der Umgebung der Blase oder mit Schleimhautlappen den Defekt überbrücken;

2. in die Methoden der direkten Vereinigung der Blasenränder nach Mobilisierung derselben; 
3. in die Methoden, welche die Exstirpation der Blasenschleimhaut und Einpflanzung der Ureteren in die Penisrinne oder in den Darmkanal bezwecken.

Jean Roux war der erste, der durch Lappenplastik einen Blasendefekt zur Heilung zu bringen suchte. Er bildete aus der Skrotalhaut einen großen Lappen und vernähte ihn, mit der Epidermisseite der Blase zugekehrt, mit den Defekträndern der Haut. Diese Methode hat dann viele Umwandlungen durchgemacht und man kann fast sagen, daß jeder Operateur seine eigene Methode ausbildete. Als Grundprinzip diente jedoch stets die Benutzung eines gestielten Lappens, der über den Defekt gelegt wurde. Manchmal wurden mehrere, bis vier Lappen entnommen, die dann gedoppelt, mit den Wundflächen einander zugekehrt, als Vorderwand der Blase benutzt wurden. Aber diese Methode zeigte bald sehr große Nachteile. Schon nach kurzer Zeit kamen die Patienten zurück mit Inkrustation und Steinbildung in der Blase, deren Ausgangspunkte die Haare bildeten. Diesem Übel. stand wurde durch Thiersch Abhilfe geschaffen, der die Bildung des doublierten Lappens verwarf und den Spalt durch zwei seitliche Brückenlappen deckte, deren jeder so groß genommen wurde, als ob er allein den Defekt decken sollte. Thiersch hatte recht gute Erfolge zu verzeichnen und seine Methode wurde später von zahlreichen Operateuren übernommen.

Im Sommer vorigen Jahres hatten wir Gelegenheit, vier von Thiersch operierte Fälle nachzuuntersuchen. Die Resultate werden in einer Dissertation veröffentlicht, ich möchte deshalb nur ganz kurz darüber berichten.

Fall I. K. Zsch., 51 Jahre alt, Bauhilfsarbeiter, wurde als 6jähriger Junge am Ende der 6oer Jahre des vorigen Jahrhunderts operiert. Die Behandlungsdauer zog sich mehrere Jahre hin, der Defekt konnte vollständig gedeckt werden. I 874 und 1878 traten Blasenbeschwerden auf und es mußten beide Male Steine operativ entfernt werden. Danach bestand völlige Beschwerdefreiheit bis zum Sommer 1912. Da stellten sich wieder Schmerzen in der Blasengegend ein. Der Urin wurde blutig. Der hinzugezogene Arzt stellte eine Geschwulst in der Blasengegend fest, deren vollständige operative Beseitigung unmöglich war. 3 Monate nach der Operation trat der Exitus letalis ein. Die mikroskopische Untersuchung des excidierten Tumors ergab Carcinom der Blase. 
Fall 2. M. St., 27 Jahre alt, Formengießer. Operiert als 5jähriger Knabe anfangs der goer Jahre. Auch diese Operationen zogen sich über mehrere Jahre hinaus. Es wurde fistellose Heilung erzielt. Bis zum Jahre 1907 bestand vollständige Beschwerdefreiheit. Damals stellten sich Blutungen aus der Harnröhre, Fieber und Schüttelfröste ein und es mußte Aufnahme ins Krankenhaus erfolgen. Es wurde eine Cystitis und Pyelonephritis festgestellt, jedoch konnte schon nach I4 Tagen der Patient wieder als geheilt entlassen werden. Er war dann wieder vollkommen beschwerdefrei, konnte sein Handwerk als Formengießer gut verrichten bis zum Jahre 1912. Damals traten Schmerzen in der linken Nierengegend auf, es ging ein erbsengroßer Stein ab, doch trat auch dann keine Besserung ein. Ende Dezember I9I2 muBte Aufnahme ins Krankenhaus erfolgen. Hier wurde eine Cystitis und Vereiterung der linken Niere festgestellt. Die Niere wurde freigelegt; sie war stark verwachsen. Es befand sich ein Stein im vorderen Teil des Ureters. Das Parenchym der Niere war stark geschrumpft und enthielt mehrere kleine Abszeßhöhlen. Im März 1913 konnte der Patient geheilt entlassen werden. Auch die Cystitis war zum größten Teil behoben, wie eine cystoskopische Untersuchung der Blase ergab. Das Fassungsvermögen der Blase betrug ca. Ioo ccm. Der rechte Ureter spritzte rhythmisch und kräftig, der Urin war klar. Der linke Ureter spritzte gar nicht.

F all 3. H. Schm., 29 Jahre alt, wurde als 8 jähriger Junge anfangs der goer Jahre operiert und nach I Jahr entlassen mit einer Fistel an der Peniswurzel. Es wurde in späteren Jahren mehrfach der Versuch gemacht, die Fistel zu schließen, jedoch ohne Erfolg. Die Sommer I9I 3 vorgenommene Nachuntersuchung ergab: Es bestanden öfters Blasenbeschwerden und Abgang von Steinen ist mehrfach beobachtet worden. Schmerzen in der Nierengegend sind nicht vorhanden. Im allgemeinen ist das Befinden ein gutes. Der Urin wird in einem Urinal aufgefangen; er ist von alkalischer Reaktion und etwas trüb, enthält $1 / 4$ pro Mille Eiweiß, keine Zylinder. Im mikroskopischen Bilde sind zahlreiche Zelltrümmer zu sehen, viel amorphe Phosphate und zahlreiche Leukocyten. Die Fistel an der Wurzel des Penis zeigt sich als ein ca. $3 \mathrm{~cm}$ breiter Spalt. Die von der Operation herrührenden Narben sind reaktionslos. Die Skrotalhaut ist leicht ekzematös.

F a 11 4. H. Kl., 4o Jahre alt, Drechsler, wurde als 2 I jähriger junger Mann anfangs der goer Jahre operiert. Auch hier betrug die Behandlungsdauer mehrere Jahre. Es trat fistellose Heilung ein. Die im Sommer I913 vorgenommene Nachuntersuchung ergab, da $B$ der jetzt 4ojährige Mann im ganzen beschwerdefrei ist. Nur im Frühjahr bestehen mehrere Tage lang Schmerzen in der Blasengegend, für die keine Erklärung gegeben wird. Es sind keine Schmerzen oder Druckempfindlichkeit in der Blasen- oder Nieren- 
gegend vorhanden. Kl. macht jeden 2. Tag eine Spülung der Blase mit abgekochtem Wasser und führt sich dazu selbst den Katheter ein. Durch diese Spülungen werden häufig grauweiße, weiche Konkremente fortgespült. Er ist kinderlos verheiratet, hat angeblich Erektionen, aber keine Ejakulationen.

Wir sehen also, daß durch die Thierschsche Operation der Zustand im großen und ganzen wohl ein leidlicher ist, daß aber cystitische Beschwerden und Konkrementbildung nicht vermieden werden können. In einem Falle trat sogar eine linkseitige abszedierende Pyelonephritis ein. Es mußten die Niere freigelegt und Abszesse incidiert werden. Die Steinbildung ist darauf zurückzuführen, daß kein vollständiger, aus Blasenschleimhaut bestehender Hohlraum geschaffen wird. Die Vorderwand der Blase wird vielmehr durch Narbengewebe ersetzt. Dadurch kann sich die Blase bei Anfüllung mit Flüssigkeit nicht zu einer Kugel ausdehnen, sondern bleibt immer ein auf dem Querschnitt mondsichelförmiger Spaltraum, in dessen Buchten und Taschen etwas Urin zurückbleibt, der sich zersetzt und Konkremente bildet. Die Narbe wird dann leicht geschwürig, schmerzhaft und macht das Tragen eines bruchbandartigen Kompressoriums unmöglich.

Die direkten Methoden haben das Bestreben, einen vollkommen mit Blasenschleimhaut ausgekleideten Hohlraum zu bilden. Der erste, der einen derartigen Versuch machte, indem er einfach die Ränder der Blasenschleimhaut anfrischte und vernähte, war Gerdi im Jahre I843. Er hatte keinen Erfolg. Auch Billroth und Rigaut gelangten nicht zu einem Resultate, da die Nähte immer wicder auseinandergingen. Wymann gelang zuerst die Vereinigung der Blasenränder, aber erst nachdem er die Blasenschleimhaut mobilisiert hatte. Katz konnte I7 Fälle sammeln, die nach der ursprünglichen Methode von Gerdi mit kleinen Abänderungen operiert worden sind. Von diesen 17 Fällen sind 9 geheilt, 5 gestorben und bei 3 gingen die Nähte wieder auseinander. Diese Methode hat auch noch den Nachteil, daß das Blasencavum ein $\mathrm{zu}$ geringes ist.

Da sie erkannten, daß man auf diese Weise nicht zum Ziele gelangen werde, weil die weit auseinanderstehenden Schambeinäste eine direkte Vereinigung der Blasenränder zu sehr hinderten, so versuchten $\mathrm{Dem}$ e und Passavant die Schambeine durch 
Druckapparat und Verbände zusammen zu bringen; sie hatten jedoch wenig Erfolg, und erst Trendelenburg blieb es beschieden, eine Methode ausfindig zu machen, die in ausgezeichneter Weise die direkte Vereinigung des Defektes ermöglicht.

Trendelenburg durchtrennt in der I. Operation die Synchondrosis sacroiliaca. Darnach lassen sich die Schambeine bei seitlichem Druck auf die Beckenschaufeln zusammenbringen, was durch eine rektale Untersuchung festgestellt werden kann. Durch Einbettung in einen Lagerungsapparat wird diese Annäherung der Schambeine zu erhalten gesucht, bis das Becken wieder fest geworden ist. Erst dann erfolgt in der 2. Operation die Anfrischung und direkte Vereinigung des Defektes womöglich in einer Etage. Auch nach dieser Operation wird das Kind noch in den Lagerungsapparat eingebettet, doch sollen jetzt weniger die Beckenhälften zusammengedrückt, als vielmehr das Kind in Rückenlage festgehalten werden. Die 3. Operation, mit der man wieder wartet, bis das Kind sich völlig erholt hat, hat den Zweck, zurückgebliebene Fisteln zu schließen. Das Verfahren Trendelenburgs, das auch verschiedentlich modifiziert wurde (vertikale Osteotomie [Berg], Osteoklasie [Koch, Kocher]), wurde zunächst von vielen Seiten übernommen, dann aber wieder verlassen, da man vor der Durchtrennung der Synchondrosen zurückschreckte. Man fürchtete auch, daß dadurch der Gang der Patienten beeinträchtigt würde. Es wurden zunächst von Czerny, Mikulicz, Schlange, König, Rydgier, Hoef t. mann, Beck u. a. Modifikationen des Verfahrens der direkten Nahtvereinigung angegeben, denen gemeinsam ist, daß sie die Vereinigung der Spaltränder ohne Synchondrosentrennung, durch besondere, die zu vereinigenden Teile mobilisierende Maßnahmen an den Rändern der Bauch- und der Beckenspalte anstrebten. Alle diese Verfahren blieben in ihren Erfolgen hinter denen Trendelenburgs weit zurück und dürften neuerdings wohl nicht mehr in Anwendung gezogen werden.

Da die direkten Methoden also nicht die von ihnen erwarteten Resultate geboten hatten und man außerdem vor der Durchtrennung der Synchondrosen zurückschreckte, verzichteten viele darauf, eine normale Blase wieder herzustellen und schlugen bei der Behandlung der Blasenspalte ganz andere Wege ein. Die 
vorgewölbte Blase als der Sitz großer Beschwerden wurde exstirpiert und die Ureteren wurden zum Teil in die Harnröhre (Sonnenburg), oder in die Bauchhaut (Harrison, Wilms, Rovsing), oder auch in den Darmkanal eingepflanzt.

Die Ableitung des Urins in den Darmkanal wurde in den 5 oer Jahren des vorigen Jahrhunderts von J. Simon und $\mathrm{J}$. R o ux inauguriert, jedoch gebührt Maydel das Verdienst, ein brauchbares Verfahren gefunden zu haben. Er führte dies zum ersten Male aus bei einem 20 jähr. jungen Mann, bei dem er die Blase bis auf ein ellipsenförmiges Stück um die Harnleiter herum excidierte und dieses in einen Längsschlitz der Flexura sigmoidea einnähte. Besonderer Wert ist auf die Erhaltung der Ureterenmündung zu legen. Die Implantation der Ureteren allein in den Darm hat sowohl im Tierexperiment als auch beim Menschen (Rein, Duplay) schlechte Resultate ergeben. Die aufsteigende Niereninfektion blieb nicht aus. Die Maydelsche Operation erfreut sich einer ziemlich ausgedehnten Anwendung, jedoch ist die Mortalität sehr beträchtlich. Enderlen konnte im Jahre I9I I in I49 Fällen eine Mortalität von über 29 Proz. feststellen. Nach ihm sind als Todesursachen zum größten Teile der Operation zur Last zu legen: Jodoform, Blutungen, Peritonitis. Im weiteren Verlauf spielt die aufsteigende Niereninfektion die Hauptrolle. Sie führt nach Jellineks und Enderlens Zusammenstellung in ungefähr 25 Proz. der Fälle zum Tode. Noch 3 oder 4 Jahre nach der Operation werden sie als Causa mortis angegeben. Unangenehme Folgen sind ferner: Intoleranz der Rektalschleimhaut gegen den Urin, Erweiterung des Rektums, Insuffizienz des Analsphinkters. Die Einpflanzung der Ureteren in das Rektum, die den Vorzug hat, daß sie extraperitoneal ausgeführt werden kann und daher eine peritonitische Infektion vermeiden läßt, hat trotzdem überraschend hohe Mortalität, so daß man annehmen möchte, daß bei der vermehrten Kotstauung im Rektum die Infektionskeime leichter Gelegenheit haben, in die Ureteren hinauf zu wandern.

Durch die Methoden, welche den Urin in das Darmrohr ableiten, wird zwar die Inkontinenz beseitigt; sie haben aber eine hohe Operationsmortalität. Außerdem ist die Gefahr der aufsteigenden Pyelonephritis eine sehr große. Daneben besteht 
noch eine andere Infirmität dieser Methoden, worauf Trendelenburg immer wieder mit Recht hingewiesen hat. „Die Urinentleerung durch den Anus nach Art der Vögel und Amphibien ist und bleibt für den Menschen nicht nur ein unnatülicher $\mathrm{Zu}$ stand, sondern auch, wenigstens für das männliche Geschlecht, eine recht unangenehme Infirmität." Das sind die eigenen Worte Trendelenburgs. Dieser Grund mag sicherlich für ihn mit ausschlaggebend gewesen sein, durch Schaffung einer normalen Blase normale Verhältnisse wieder herzustellen.

Ich möchte nun zunächst alle bisher von Trendelenburg selbst oder nach seiner Methode von anderen Operateuren veröffentlichten Fälle bringen und dann noch eine Reihe von neuen hinzufügen.

\section{A. Frühere Fälle.}

Fall I. Operateur: Trendelenburg. Ernst Rabe, 4 Jahre alt, I88I. Es ist eine vollständige Bauch-Blasen-Genitalspalte vorhanden. Die Entfernung der Schambeine beträgt $6 \mathrm{~cm}$. In der ersten Operation wird die Trennung der rechten Synchondrosis sacroiliaca vorgenommen, danach lassen sich bei seitlichem Druck auf beide Beckenhälften die Schambeine fast zusammenbringen. In derselben Sitzung wird die Anfrischung und Naht der Spaltränder von Blase und Penis vorgenommen. Darüber wird die Haut des Bauches und der Wurzel des Penis von den Seiten her zusammengezogen und in der Mittellinie durch zwei Reihen von Nähten vereinigt. Durch die neugebildete Harnröhre wird ein Drain in die Blase eingeführt. Das Kind wird in einen Apparat gelegt, der zwei seitliche Pelotten hat, mit denen von außen ein Druck auf die Trochanteren ausgeübt werden kann. Exitus letalis am i8. Tage.

Die Sektion ergibt: Erweiterung beider Ureteren und beider Nierenbecken. Abszedierende Nephritis.

Fall 2. Operateur: Trendelenburg. Es handelt sich um einen $21 / 2$ jährigen Knaben, bei dem eine vollständige Bauch-BlasenGenitalspalte besteht. Der Abstand der Schambeine beträgt $5 \mathrm{~cm}$.

Am 4. VIII. I 885 werden beide Synchondrosen durchtrennt. Das Kind wird in einen Lagerungsapparat eingebettet. Die Wunde heilt per primam.

Die nächsten Operationen finden am I3. X. und 25. XI. I885 statt. Die Spaltränder werden angefrischt und in zwei Etagen genäht. Diese Operationen haben den Erfolg, daB alles verheilt ist bis auf eine Fistel an der Blase und eine am Infundibulum. Nach Verlauf eines Jahres wird der Versuch gemacht, die Fisteln zu schließen. In ausgiebiger Weise wird eine Ablösung des Blasenhalses und des 
hinteren Teiles der Harnröhre von den Schambeinen vorgenommen, um die seitliche Spannung zu beseitigen. Die Wunde wird mit Jodoformgaze tamponiert. Es tritt eine Jodoformintoxikation ein und das Kind stirbt.

F all 3. Operateur: Trendelenburg. E. B., 21/2 Jahre alt.

Es besteht eine vollständige Bauch-Blasen-Genitalspalte. Der Abstand der Schambeine beträgt 5--6 cm.

Am 9. II. I 886 werden beide Synchondrosen durchtrennt.

In einer zweiten Operation am I2. III. wird die Anfrischung der Spaltränder und Naht der Blase mit Silberdraht in einer Etage vorgenommen.

Resultat: Nur im oberen Teile ist eine primäre Vereinigung eingetreten.

Am 20. IV. wird die Operation wiederholt. Die Spaltränder werden breit angefrischt und durch Silbernähte vereinigt.

Resultat: Prima intentio, bis auf eine kleine Fistel in der Mitte der früheren Blasenspalte.

Am 25. V. wird diese Fistel geschlossen und die Ablösung der Harnröhre im Bereich des Penis durch Anfrischung und Naht in einer Etage vorgenommen.

Die Fistel öfnet sich wieder. Die Naht des Penis heilt per primam bis auf zwei haarfeine Fisteln an der Wurzel des Penis.

In einer weiteren Operation werden diese Fisteln geschlossen. Da der hintere Teil der Harnröhre und der Blasenhals noch sehr weit sind, wird gleichzeitig eine Verengerung vorgenommen dadurch, daß auf jeder Seite ein Streifen Urethralhaut fortgenommen wird. Diesmal Heilung per primam.

Ergebnis der Operation: Das Kind wird am 9. VIII. I889 mit elastischem Beckengurt und Urinal entlassen.

Die Fistel der früheren Blasenspalte ist später von $\mathrm{M} \mathrm{ikulicz}$ geschlossen worden. Über das weitere Ergehen des Kindes berichtet $1897 \mathrm{Dietze}$ : Es hat sich bei ihm in fast normaler Weise die Kontinenz wieder hergestellt.

Fal1 4. Operateur: Trendelenburg. Bei dem I $1 / 2$ jährigen Knaben G. G. ist die Blase sehr klein, mit papillären Exkreszenzen bedeckt. Sie läßt sich infolgedessen nicht zu einem Cavum einstülpen und durch direkte Naht vereinigen. Die Durchtrennung der Synchondrosen wird trotzdem im Oktober 1886 vorgenommen, um dadurch sowohl für die normale Gestaltung des Penis, als auch für die erforderliche Deckung des Blasenspaltes durch Lappen günstigere Bedingungen zu schaffen. Die Operationswunde heilt per primam. In der zweiten Operation wird die Bildung des Penis durch Anfrischung und Naht vorgenommen und die Deckung des Defekts durch Lappenbildung aus der Bauchhaut. Es heilt alles bis auf einen Spalt an der rechten Seite des Lappens, durch den sich zeitweilig der 
Ureter vordrängt. Wegen schlechten Allgemeinzustandes wird von weiteren Operationen Abstand genommen. Bei der Entlassung kann das Kind bereits wieder einige Schritte gehen. Die letzten Nachrichten stammen aus dem Jahre I90I. Der jetzt I6jährige junge Mann befindet sich wohl, liegt nachts immer trocken, wacht zwei- bis dreimal mit Harndrang auf, läßt Urin und schläft weiter. Im Stehen kann er den Urin nur mit großer Willensanstrengung für kurze Zeit halten, trägt deshalb ein Urinal, mit dem er den Harn 2 Stunden, zurückhalten kann. Das Tragen des Apparats macht Beschwerden.

Fall 5. Operateur: Trendelenburg. Bei einem 5jährigen Mädchen besteht eine vollständige Bauch-Blasen-Genitalspalte. Der Abstand der Schambeine beträgt $5 \mathrm{~cm}$. Als Komplikation findet sich ein Nabelbruch.

Am 6. VI. 1886 werden beiderseits die Synchondrosen durchtrennt. Der Heilungsverlauf ist ein ungestörter.

Am 9. bis 13. VI. wird der Verschluß der Spaltränder in derselben Weise vorgenommen wie bei dem Knaben E. B. (Fall Nr. 3). Dabei wird der Nabelbruch, dessen Oberfläche mit in die Anfrischung hinein fällt, radikal operiert. Nach 8 Tagen ist alles verheilt bis auf eine erbsengroße Fistel in der Mitte der Blase, deren Verschluß durch Anfrischung am 3I. I. I 887 vorgenommen wird. Gleichzeitig wird eine Verengerung der hinteren Urethra vorgenommen. Die Fistel bricht wieder auf und wird durch einen zungenförmigen Lappen aus der Bauchhaut zum Verschluß gebracht. Diesmal tritt Heilung ein, aber es besteht völlige Inkontinenz. Es wird dann nochmals ein Versuch gemacht, durch künstliche Verengerung der Urethra Kontinenz zu erzielen. Nach Spaltung des Mons veneris zeigt es sich, daß die Urethra durch das Auseinanderfedern beider Schambeine zu einem breiten Spalt auseinander gezogen wird. Um die dadurch bedingte Spannung zu beseitigen, wird eine nochmalige Synchondrosentrennung vorgenommen und dann die Urethra vereinigt. erzielt.

Resultat: Auch durch diese Operation wird keine Kontinenz

Letzte Nachricht aus dem Jahre I90I: Allgemeinzustand gut. Kontinenz nicht vorhanden.

Fall 6. Operateur: Trendele n burg. Bei einem $4^{3 / 4}$ Jahre alten Knaben besteht vollständige Bauch-Blasen-Genitalspalte.

Am 23. IX. 1890 wird auf beiden Seiten die Synchondrosentrennung vorgenommen. Die Wunde heilt nicht ganz primär.

In einer zweiten Operation am 9. I. I89 I erfolgt die Anfrischung und Naht der Spaltränder, wie in Fall 3, mit möglichster Verengerung des hinteren Abschnittes der Urethra. Durch den oberen Wundwinkel wird in die Blase ein feines Glasröhrchen eingelegt. Das Kind wird in den Lagerungsapparat eingebettet. 2 Tage nach der Operation ent- 
leert das Kind, als sich das Drain verstopft, den Urin in kräftigem Strahle alle 20 Minuten aus der Blase. Kurz vor der Urinentleerung fängt der Knabe jedesmal an zu weinen, dann ruft er: es kommt, es kommt, und nun spritzt der Urin in ganz normaler Weise aus der Harnröhre hervor. Leider erfolgt die Heilung der Wunde nicht per primam. Es bilden sich 3 Fisteln, die eine am Penis, die anderen in der Mitte der vorderen Blasenwand. Diese werden am I4. III. teils durch Anfrischung und direkte Naht, teils durch Lappenbildung zum Verschluß gebracht.

Resultat: Die Fistel am Penis ist wieder aufgebrochen.

Bei der Entlassung am I2. IV, läuft im Stehen sämtlicher Urin aus dieser Fistel ab. Im Liegen kann der Harn $3 / 4$ Stunde zurückgehalten werden. Die letzten Nachrichten stammen aus dem Jahre I90I. Der Allgemeinzustand ist ein guter, es besteht vollkommene Inkontinenz.

Fall 7. Operateur: Trendelenburg. Es handelt sich um einen 6jährigen Knaben mit vollständiger Bauch-Blasen-Genitalspalte. Durch 5 Operationen wird die Heilung des Defekts erzielt, wobei die Operationen, die zur Beseitigung der beiden angeborenen Brüche dienten, nicht mit gerechnet sind.

In der ersten Operation wird die Durchtrennung der rechten Synchondrosis sacroiliaca vorgenommen. Beide Schambeine lassen sich aneinander bringen, so daß die linke Synchondrosis nicht durchtrennt zu werden braucht. Das Kind wird in den Lagerungsapparat gebracht. Der Urin wird durch eine Bunsensche Wasserstrahlpumpe abgesaugt, wodurch bewirkt wird, daB das Kind immer trocken liegt und die Operationswunde nicht mit Urin durchtränkt wird. Heilung der Operationswunde per primam. 3 Monate später, nachdem das Becken wieder fest geworden ist, wird in Beckenhochlagerung die Anfrischung und Naht der Spaltränder in einer Etage vorgenommen. In der Mitte der vorderen Blasenwand wird zwecks Ableitung des Urins eine kleine öffnung gelassen, durch die ein Drain in die Blase eingeführt wird. Der Urin wird durch die Wasserstrahlpumpe abgesaugt. Es tritt prima intentio ein bis auf eine Fistel am Blasenhals.

In einer 3. Operation wird die absichtlich in der vorderen Blasenwand zurückgelassene öffnung durch Lappenplastik verschlossen, wobei wiederum eine kleine Fistel am Scheitel der Blase zurückbleibt.

Die beiden Fisteln werden durch Lappenbildung, Umschneidung und Naht in einer 4. Operation zum Verschluß gebracht. Dabei werden Blasenhals und hinterer Teil der Urethra in ausgiebiger Weise von den Schambeinen abgelöst und, ,da diese nach dem Aufklappen der Rinne als abnorm weit erscheint, so daB zu befürchten ist, der durch Naht geschlossene Ring möchte zu weit werden, um einen Verschluß des Halses durch Sphinkterwirkung zustande kommen zu lassen", wird vor Anlegung der Naht vom Rand der Blase und 
Urethralrinne ein schmaler Streifen fortgenommen. Der Urin wird durch einen dünnen Katheter abgeleitet. Bis auf eine haarfeine Fistel tritt primäre Heilung ein. Diese Fistel wird durch einen weiteren operativen Eingriff leicht geschlossen.

Resultat: Das Kind kann den Urin 2 Stunden zurückhalten. Die Menge des im kräftigen Strahl entleerten Urins beträgt $40 \mathrm{ccm}$ und darüber. Der Urinstrahl kann willkürlich abgebrochen und wieder in Gang gesetzt werden, so daß die Funktion der Blase also vollständig wieder hergestellt ist. Der einzige noch vorhandene geringfügige Mangel besteht darin, da $B$ das Urinieren alle zwei Stunden, also häufiger als normal, erfolgt und daß bei psychischer Erregung mitunter einige Tropfen Urin unwillkürlich abgehen. Für gewöhnlich bleiben die Kleider ganz trocken. Nachts muß das Kind einige Male zum Urinlassen geweckt werden, wenn es trocken bleiben soll. Diese Fähigkeit, den Urin zu halten, ist später wieder verloren gegangen. Das Kind hat einen Apparat bekommen, mit dem dic Harnröhre leicht komprimiert und dadurch der Urin stundenlang zurückgehalten werden kann.

Die letzten Nachrichten stammen vom November 1913, durch ausführliche briefliche Mitteilung des Vaters. Der Allgemeinzustand ist ein guter. Der Urin kann ohne Apparat nur etwa ro Minuten gehalten werden, mit Apparat kann er bis zu 6 Stunden zurückgehalten werden; nachts kann durchgeschlafen werden.

Fall 8. Operateur: Küster. Wilhclm D., 45 Jahre alt.

Es handelt sich um eine vollständige Bauch-Blasen-Genitalspalte. Der Abstand der Schambeine beträgt $7,5 \mathrm{~cm}$.

Am 28. I. I 89 I wird die linke Synchondrosis durchtrennt. Die Operationswunde infiziert sich, es bildet sich ein großer retroperitonealer Abszeß, der von einer Laparotomie aus incidiert und drainiert wird. Der Exitus letalis tritt am 24. II. ein.

Fall 9. Operatcur: Küster. Bei cinem r ı jährigen Knaben besteht vollständige Bauch-Blasen-Genitalspalte. Der Abstand der Schambeine beträgt $6 \mathrm{~cm}$.

Am I I. XII. I 890 werden beide Synchondrosen durchtrennt. Die Wunde wird mit Jodoformgaze drainiert. Wieder erfolgt eine Infektion der Operationswunde. Der Absze $B$ wird eröffnet und drainiert von Incisionen in der Inguinalgegend aus, worauf sich das Kind langsam erholt. Zur weiteren Kräftigung wird es im Juli I890 nach Hause entlassen. Bei der Wiederaufnahme im September I89I beträgt der Abstand der Schambeine $2 \mathrm{~cm}$.

Am I5. IX. wird die Anfrischung und Naht der Spaltränder vorgenommen. Da sehr starke Spannung vorhanden ist, werden die horizontalen und absteigenden Schambeinäste durchmeißelt und die Symphyse mit Draht genäht. Bis auf eine Fistel am Blasenhals heilt die Wunde per primam. 
Diese Fistel wird am 12. XI. I89I angefrischt und genäht. Zu gleicher Zeit erfolgt die Bildung der Urethra. Die Fistel bricht von neuem auf und es sind noch vier operative Versuche nötig, um die Fistel zu schließen.

Resultat: Der Defekt ist vollkommen geheilt. Bei der Entlassung kann der Urin 20 Minuten gehalten werden.

Im folgenden Jahr wird eine Nachuntersuchung vorgenommen. Sie ergibt, daß der Urin einige Zeit gehalten und dann im Strahl entleert werden kann.

F all ro. Operateur: Küster. Bei dem 7jährigen $H$. Z. besteht eine vollständige Bauch-Blasen-Genitalspalte. Der Abstand der Schambeine beträgt $5 \mathrm{~cm}$.

Am I9. VI. I 890 wird dic Bildung der Harnröhre nach dem Verfahren von $\mathrm{Th}$ ier $\mathrm{s}$ h vorgenommen. Am 5. XII. desselben Jahres erfolgt dic Durchtrennung der linken Synchondrosis, die erst nach größter Anstrengung gelingt. Die Schambeine können jedoch nicht vollständig in Berührung miteinander gebracht werden. Die Operationswunde wird tamponiert und offon behandelt. In den nächsten Tagen stellt sich Fieber ein, es bildet sich ein großer Abszeß in der linken Leistengegend, der durch eine Incision oberhalb des Ligamentum Pouparti eröffnet und drainiert wird. Darauf tritt Besserung des Zustandes ein, doch erholt sich das Kind nicht wieder. Es siecht vielmehr langsam dahin und 4 Monate nach der Operation tritt Exitus letalis ein.

Die Sektion ergibt: Allgemeine Miliartuberkulose.

Fall II. Operateur: Brockmann. Bei cinem 4jährigen Knaben mit vollständiger Bauch-Blasen-Genitalspalte wird eine Durchtrennung der Synchondrosen beiderseits vorgenommen. Darauf werden die Spaltränder der Blase angefrischt und genäht.

Resultat: Vollständige Heilung des Blasendefekts. Keine Kontinenz. Die Epispadie wird nicht behandelt.

F a 11 I2. Operateur: Makins. Es handelt sich um einen 5jährigen Knaben. Zunächst werden beide Synchondrosen durchtrennt. 2 Monate darauf wird die Anfrischung und Naht der Spaltränder der Blase vorgenommen. Die Naht hält nicht, worauf die Deckung durch einen seitlichen Bauchdeckenlappen erfolgt.

Fall I3. Operateur: Duret (Lille). Es handelt sich um ein 9jähriges Mädchen, das 1897 operiert wurde. Zunächst werden beide Synchondrosen durchtrennt. Dann wird ein Schnitt entlang dem Schleimhautrande der Blase geführt. Die Blasenränder werden abgelöst und genäht. Der Spalt in den Bauchdecken wird durch zwei seitliche Lappen gedeckt. Es tritt vollkommene Heilung ein.

Ergebnis: Das Fassungsvermögen der Blase beträgt I Jahr später $60 \mathrm{ccm}$. 
Die letzten Nachrichten stammen aus dem Jahre I896. Damals ist die Patientin an einer Lungentuberkulose gestorben.

Fall I4. Operateur: Rydgier (Krakau). Es handelt sich um ein kleines Kind mit vollständiger Bauch-Blasen-Genitalspalte. Die Durchtrennung der Synchondrosen und Anfrischung der Blase wird in einer Sitzung vorgenommen. Darauf wird das Kind in den Lagerungsapparat eingebettet. Das Kind geht an einer Infektion der Operationswunde zugrunde.

F all I5. Operateur: Rydgier. Bei einem Kind mit vollständiger Bauch-Blasen-Genitalspalte wird zunächst die Durchtrennung der Synchondrosen vorgenommen. Die Wunden werden durch Nähte geschlossen, darauf das Kind in Bauchlage gebracht, bis primäre Heilung der Operationswunden eingetreten ist. Dann erst wird das Kind in den Lagerungsapparat cingebettet. genäht.

In einer 2. Operation werden die Spaltränder angefrischt und

Ergebnis: Nur im oberen Teile hat die Naht gehalten, im unteren ist sic auseinander gegangen.

Fall 16. Operateur: Delagén ière. Bei einem sehr kleinen Kind wird im Jahre 1894 die Operation nach Trendelenburg: vorgenommen. Es gelingt, einen Teil des Defekts zu schließen. Das Kind wird zur Erholung nach Hause geschickt und geht dort an Diarrhöe zugrunde.

Fall 17. Operateur: D cla gén ière. Bei einem fast 2jährigen Kinde wird im Jahre 1899 die Durchtrennung der Synchondrosen vorgenommen. Noch am Abend des Operationstages erfolgt der Exitus letalis.

Fall 18. Operateur: Delagénière stellt auf dem internationalen Kongreß für Medizin (Sektion: Chirurgie; Montagsitzung; am 6. VIII. I900) ein I Ijähriges Kind vor, bei dem er eine vollständige Bauch-Blasen-Genitalspalte nach dem Verfahren von Trendelenbu rg operiert hat. Die Operation erstreckte sich auf einen Zcitraum von 7 Monaten, bis vollständige Heilung eintrat. Vollständige Kontinenz. Normale Sphinkterfunktion.

Letzte Nachricht vom Juli 1902: Das Kind ist noch jetzt, 13 Jahre nach der Operation, vollständig kontinent.

\section{B. Bisher noch nicht veröffentlichte Fälle.}

Fall i9. Operateur: Trendelenburg. Bei einem 3jährigen Knaben mit gesunden inneren Organen besteht eine vollkommene Bauch-Blasen-Genitalspalte. Der Abstand der Schambeine beträgt $5 \mathrm{~cm}$. Es ist beiderseits eine Skrotalhernie vorhanden. Die Heilung der Blasenspalte wird erreicht durch im ganzen 7 Operationen, wobei die Operation zwecks Beseitigung des rechten sehr groBen 
Skrotalbruches nicht mit gerechnet ist. Die Behandlungsdauer zieht sich ca. 3 Jahre hin. Das Kind hat mehrfach fieberhafte Anginen durchzumachen mit sehr starker Reizung der Nieren, die es sehr herunter bringen. Dann bekommt es noch Diphtherie und später auch Masern. Infolgedessen ist zwischen den einzelnen Operationen oft ein längerer Zwischenraum.

In der I. Operation wird die Durchtrennung beider Synchondrosen vorgenommen. Das Kind wird in den Lagerungsapparat cingebettet, der Urin mittels der Wasserstrahlpumpe abgesaugt. Heilung der Wunde per primam.

In einer 2. Operation wird in Beckenhochlagerung die Anfrischung und Naht der Blase vorgenommen. In der Mitte der Blasenwand wird eine Öffnung zur Ableitung des Urins gelassen. Die Haut wird nicht über der Blase zusammengezogen. Das Kind wird wieder in den Apparat gelegt. Die Absaugung des Urins erfolgt durch die Wasserstrahlluftpumpe. Der Heilungsverlauf ist cin ungestörter.

Durch eine 3. Operation erfolgt der Verschlub der absichtlich an der Blase gelassenen öffnung durch Anfrischung und Naht. Gleichzeitig wird die Urethralrinne gebildet. Da sich die Haut nicht zusammenziehen läßt, wird auf der linken Seite ein größerer Bauchdeckenlappen gebildet, der in die angefrischten Hautränder eingenäht wird mit Ausnahme einer Stelle auf der linken unteren Seite. Hier wird eine öffnung gelassen zur Ableitung des Urins und ein Drain in die Blase cingeschoben.

Diese Öfnung und eine Fistel am unteren Teile des Lappens werden in einer 4. Operation teils durch Anfrischung und direkte Naht, teils durch Lappenbildung geschlossen. Wiederum bildet sich eine etwa bohnengroße Fistel an der Wurzel des Penis, sie nimmt spaltförmige Gestalt an. Auch der hintere Teil der Urethralrinne liegt wieder frei.

In einer 5. Operation wird nochmals die rechte Synchondrosis sacroiliaca durchtrennt, dann wird noch ein Schnitt in der Rhaphe des Skrotums gemacht, der die Symphyse freilegt. Die noch knorpeligen Schambeinäste werden durchtrennt und die Symphyse wird mit Draht genäht. Die Operationswunden werden nun zum Teil genäht, zum größten Tcil tamponiert. Das Kind wird in den Lagerungsapparat eingebettet, der Urin durch die Wasserstrahlpumpe abgesaugt. Der Wundverlauf ist ein ungestörter.

In 2 weiteren Operationen werden die Fisteln zum Verschluß gebracht und auch die hintere Harnröhre gebildet. Besondere Sorgfalt wird wieder darauf gelegt, da $\beta$ der Blasenhals eng genug wird. Nach einiger Zeit bildet sich am Damm ein Abszeß. Der Symphysendraht ist durchgeschnitten, wird entfernt, worauf die eitrige Sekretion sofort nachläßt. 
Ergebnis: Der Defekt ist vollkommen geschlossen. Der Urin kann bis zu 1/2 Stunde gehalten werden. Das Kind kann den Urinstrahl willkürlich abbrechen und wieder in Gang setzen. Diese Fähigkeit, den Urin zu halten, hat etwa I Jahr lang bestanden und ist dann wieder verloren gegangen. Es haben sich dann cystitische Beschwerden eingestellt und es sind mehrere Blasensteine spontan abgangen.

Im Jahre 1907 wird durch Sectio alta ein pflaumengroßer Stein entfernt. Die Operationswunde heilt o. B.

Letzte Nachricht im September 1913: Der jetzt 17 Jahre alte L. G. hat sich kräftig entwickelt, betreibt das Schmiedehandwerk. Er hat nie Schmerzen in der Blasen- oder Nierengegend. Einen Apparat trägt er nicht. Der ständig tropfende Urin wird in Windeln aufgefangen. Skrotum und Hoden haben sich ziemlich normal entwickelt. Auch die Form und Größe des Penis ist befriedigend. Schamhaare sind mäßig, am wenigsten in der Medianlinie entwickelt. Dic Haut des Skrotums ist ekzematös. Die Blase wölbt sich vor. Die Operationsnarben sind reaktionslos. Wird die Aufmerksamkeit abgelenkt, so kann der Urin einige Minuten gehalten und dann im Strahl entleert werden. Der Harn ist mit weißlichen Flocken vermischt, hat alkalische Reaktion. Im mikroskopischen Bilde finden sich reichlich Zelltrümmer und Leukocyten in größerer Anzahl als normal. Die von Blase und Nieren aufgenommenen Röntgenbilder lassen einen Stein nicht erkennen. Der Symphysenspalt ist ca. drei Querfinger breit. Die Cystoskopie der Blase ergibt: Die Blase ist leicht zu füllen und hat ein Fassungsvermögen von über $\mathrm{I}$ oo $\mathrm{ccm}$. Sie ist nur wenig nach den Seiten ausgezogen und hat fast kugelige Form. Die vordere Blasenwand ist ziemlich dünn, an der Schleimhaut sind nirgends Blutungen zu sehen. Die Ureterenmündungen springen abnorm weit vor, sind auf das Doppelte erweitert und etwas gewulstet. Die Urinabsonderung erfolgt rhythmisch, in kräftigem, etwas wirbelndem Strahl. Der Urin ist klar.

Da sich herausstellt, daß durch einen leichten Druck auf die hintere Urethra der Urin sehr gut zurückgehalten werden kann und außerdem der Penis sich so weit entwickelt hat, daß sich bequem ein Urinal anbringen läßt, wird ein Apparat mit Pelotte verordnet. Damit kann der Urin gut zurückgehalten werden.

Fall 2o. Operateur: Trendelcnburg. Es handelt sich um einen 32 jährigen Arbeiter, Fr. D., bei dem eine vollständige BauchBlasen-Genitalspalte vorhanden ist. Der Abstand der Schambeine beträgt $8,5 \mathrm{~cm}$. Es wird ein vollkommener Verschluß des Defektes erzielt durch im ganzen sieben operative Eingriffe. Die Behandlungsdauer erstreckt sich über 3 Jahre.

In der I. Operation wird eine rechtseitige Osteotomie vorgenommen, worauf sich die Schambeine fast aneinander bringen lassen. 
Der Patient wird in den Lagerungsapparat gebracht, der jedoch nicht gut vertragen wird. Es treten vielmehr an mehreren Stellen Dekubitalgeschwüre auf, die aber ohne Besonderheiten abheilen. Die Operationswunde hat auch eine Zeitlang geeitert, hat sich dann aber ohne weiteres geschlossen. Nachdem die Knochenwunde wieder fest geworden ist, wird der Patient außer Bett gebracht und erlernt das Laufen schr bald wieder. Dabei stellt sich eine Lähmung des rechten $\mathrm{N}$. peronaeus und Tibialis heraus, die durch Elektrisieren allmählich gebessert wird.

In der 2. Operation wird nach Ablösung der Blasenschleimhaut von der Symphyse die Anfrischung und Naht der Blase und Harnröhre vorgenommen. Die Bauchhaut kann nicht darüber zusammengezogen werden. Dieser Defekt wird durch einen seitlichen Bauchdeckenlappen geschlossen, bis auf eine Stelle am oberen Rande, wo ein Drain in die Blase eingeführt wird, um den Urin mit der Wasserstrahlpumpe abzusaugen. Die Naht der Harnröhre heilt per primam. Die Blasennaht heilt nur in den oberen zwei Dritteln. Der Lappen heilt ein, hat aber große Neigung zu schrumpfen und rollt sich schließ. lich ganz auf. Er wird in einer 3. Operation wieder ausgebreitet und über dem Defekt vernäht und heilt jetzt gut ein. Der Verschluß des unteren Blasendrittels - - die hintere Blasenwand ist noch in etwa Fünfmarkstückgröße vorgefallen - gelingt in zwei weiteren Operationen, wobei die Blasenränder direkt vereinigt werden, während die Deckung des Hautdefekts durch Lappenplastik vorgenommen wird. Am Blasenhals ist absichtlich eine öfnung gelassen worden, durch die ein Drain zur Ableitung des Urins in die Blase eingeschoben wird. Dicse Öffnung wird durch einen seitlichen Lappen später gedeckt, der bis auf eine linsengroße Fistel an der Peniswurzel anheilt. Diese Fistel läßt sich unschwer zum Verschluß bringen.

Ergebnis: Der Defekt ist vollkommen geheilt. Der Urin kann im Liegen über $1 / 2$ Stunde gehalten und dann in kräftigem Strahle entleert werden. Der Strahl kann willkürlich abgebrochen und wieder in Gang gesetzt werden. Im Stehen tropft der Urin dauernd ab. Der Patient wird mit Urinal entlassen. Die Fistel an der Peniswurzel, die zuletzt geschlossen wurde, ist später öfters aufgebrochen, hat sich aber immer wieder spontan geschlossen. Im April 1905 öffnet sie sich von neuem und wird jetzt operativ verschlossen. Es hat damals auch eine starke Cystitis bestanden, die sich aber bald gebessert hat. Im September desselben Jahres ist Exitus letalis eingetreten.

Die Sektion (Dr. H. C e ele n, Charité Berlin) ergibt:

Lobäre Pneumonie des rechten Ober- und Mittellappens. $\mathrm{Cy}$ stitis gangraenosa und zahlreiche Erosionen der Blasenschleimhaut. Pyelonephritis abscedens. Die Nieren klein, mit zahlreichen, kleinen, gelblichen Konkrementen und vielen hydronephritischen Buchten. Die linke Niere ist total verjaucht. Herz schlaff. 
Fall 2r. Operateur: Trendelenburg. Es handelt sich um einen 4jährigen Knaben mit vollständiger Bauch-Blasen-Genitalspalte, einer doppelseitigen Leistenhernie und Kryptorchismus.

Am 26. VI. 1903 wird in ungestörter Chloroformnarkose und Beckenhochlagerung zunächst die Durchtrennung beider Synchondrosen und dann die Anfrischung und Naht der Spaltränder in einer Etage vorgenommen.

Am oberen Rande der Blase wird eine Öffnung gelassen und hier ein Drain in die Blase eingeschoben. Dann wird das Kind in den Lagerungsapparat gebracht, der Urin mit der Wasserstrahlpumpe abgesaugt. In den nächsten Tagen ist das Kind sehr unruhig, schreit viel, verweigert die Nahrungsaufnahme. Der Puls ist stark beschleunigt. Im Urin ist Eiweiß nachweisbar. Die Operationswunden über den Synchondrosen heilen per primam, während die Blasennaht auseinander zu gehen beginnt. Das Kind verfällt immer mehr.

Am 8. VII. tritt der Exitus letalis ein.

Scktionsergebnis (Marchand): Dilatatio ureteris utriusque et hydronephrosis bilateralis. Ureteritis, Pyelitis, Pyelonephritis duplex. Status lymphaticus.

Fall 22. Operateur: Trendelenburg. Es handelt sich um ein I Ijähriges Mädchen L, M., das am 27. I. I905 aufgenommen wird. Es besteht eine vollständige Bauch-Blasen-Genitalspalte. Synphysenspalt sehr breit.

Am I8. II. werden beide Synchondrosen durchtrennt und in Beckenhochlagerung wird die Anfrischung und Naht der Blase angeschlossen. Die Schambeine werden durch eine Drahtnaht zusammengehalten. Im oberen Teil der Blase wird eine Öffnung gelassen und hier ein Drain in die Blase eingeschoben. Das Kind wird nicht in den Apparat gebracht, sondern auf die Seite gelegt. Am folgenden Tage hat das Kind andauernd gebrochen.

Plötzlicher Kollaps. Exitus letalis.

Sektionsergebnis (Marchand): Dilatatio ureteris inf. sin. et pelvis renalis inf. renis sin. Cicatrices renis sin. e pyelonephritide sin. Embolia adiposa et emphysema interstitiale pulm. Hepar adiposum.

Fall 23. Operateur: Trendelenburg. Es handelt sich um einen 7 jährigen Knaben mit vollständiger Bauch-Blasen-Genitalspalte, doppelseitiger Leistenhernie.

Bei der ersten Operation am 20. I. I906 werden beide Synchondrosen durchtrennt, die Wunden genäht. Das Kind wird seitlich gelagert und die obere Beckenhälfte mit einem Sandsack beschwert. Heilung der Wunde erfolgt per primam. Es treten an mehreren Stellen Dekubitalgeschwüre auf, so daß die Belastung fortgelassen werden muß, worauf die Geschwüre abheilen. Da der Symphysenspalt noch zu groß ist, wird in einer zweiten Operation am i7. II. 
beiderseits etwa 2 Querfinger breit seitlich von der Rhaphe scrotalis eine etwa $6 \mathrm{~cm}$ lange Incision auf die absteigenden Schambeinäste zu gemacht, und diese werden am Übergang des Os ischii durchmeißelt. Darauf werden auch die horizontalen Schambeinäste etwa in der Gegend der Tubercula pubis durchtrennt. Die abgemeiBelten Stücke bleiben mit dem Periost so weit wie möglich im Zusammenhang. Da sie von den Musc. recti nach hinten gezogen werden, werden die Muskeln durchschnitten. Auf diese Weise gelingt es, die beiden Fragmente einander zu nähern. Die große Wundhöhle wird zum Teil tamponiert, zum Teil genäht. Während der Operation entleert das Kind etwas Stuhl, wodurch das Wundgebiet beschmutzt wird. Infolgedessen tritt eine Infektion der Wunde ein, dic aber gut verläuft. Die Wundfläche granuliert zu, so daß am I6. III. in einer 3. Operation die Anfrischung und Naht der Blase vorgenommen werden kann. Am Scheitel der Blase wird eine öffnung gelassen, durch die ein Drain eingeführt wird zur Ableitung des Urins. Über die neugebildetc Harnröhre wird die Penishaut von beiden Seiten herübergezogen, über der Blase werden die Bauchdecken wegen der Spannung nur zum Teil geschlossen.

Der operative Eingriff wird ausgezeichnet überstanden. Die Wunde sieht in den ersten vier Tagen gut aus. Am fünften Tage sickert der Urin tropfenweise zwischen den einzelnen Nähten durch und allmählich gehen die ganzen Nähte wieder auf. Das Kind bekommt die Masern und wird auf die Infektionsabteilung verlegt. Von dort kommt es Anfang Juli zurück. Es wird nun nach reichlicher Polsterung der Trochanterengegend eine Gummibinde um das Becken gelegt und dadurch eine seitliche Kompression ausgeübt. Die Bindenwicklung wird Tag und Nacht fortgesetzt, wird sehr gut vertragen und hat den Erfolg, daB die Blase dauernd invertiert ist und daB der Abstand der Spinae, der früher $20 \mathrm{~cm}$ betrug, bis auf $13 \mathrm{~cm}$ verringert ist. Bevor ein nochmaliger operativer Eingriff vorgenommen werden kann, wird das Kind von den Eltern nach Hause geholt.

Fall 24. Operateur: Trendelenburg. Bei dem 4jährigen Knaben W. H. ist eine vollständige Bauch-Blasen-Genitalspalte vorhanden. Der Abstand der Schambeine beträgt $4 \mathrm{~cm}$. Es wird eine vollständige Heilung erzielt. Die Behandlungsdauer zieht sich über 3 Jahre hin. Sie wird öfters dadurch unterbrochen, daB das Kind monatelang krank ist.

Die erste Operation wird am 10. VII. I 899 vorgenommen. Beide Synchondrosen werden durch bogenförmigen Schnitt freigelegt und mit dem Messer durchtrennt. Dann wird das Kind in den Lagerungsapparat gelegt mit $5 \mathrm{~kg}$ Belastung, der Urin mittels der Wasserstrahlpumpe abgesaugt. Die Heilung der Wunden erfolgt fast primär. Die Blase sinkt vollkommen zurück. Bald ist der Lagerungsapparat nicht 
mehr nötig. Schon bei seitlicher Lagerung, mit geringer Belastung der oben befindlichen Beckenschaufel durch einen Sandsack, stoßen die Schambeine zusammen, wie durch rektale Untersuchung festgestellt wird. Das Kind macht dann eine typhöse Erkrankung durch.

Am 26. X. werden in einer zweiten Operation die Spaltränder von Blase und Harnröhre angefrischt und durch Katgutknopfnähte vereinigt. In den oberen Teil der Blase wird ein Drainrohr eingeführt zur Ableitung des Urins. Die Haut wird nur zum Teil über der Blase zusammengezogen. Die Wundflächen werden tamponiert, das Kind in den Lagerungsapparat gelegt, der Urin mittels der Wasserstrahlpumpe abgesaugt. Die Wundheilung ist zunächst ungestört, dann geht der größte Teil der Blasennaht wieder auf, während die Naht des Blasenhalses und der Urethralrinne gelungen ist, bis auf eine winzige Fistel an der Corona glandis. Durch einen großen Bauchdeckenlappen, dessen Stiel in der Nabelgegend liegt, wird in einer dritten Operation der Verschluß der Blase herbeigeführt. Die Fistel selbst wird durch Anfrischung und direkte Naht geschlossen.

Ergebnis: Bei der Entlassung ist eine vollständige Heilung des Defekts zu konstatieren. Es besteht zunächst vollständige Inkontinenz, die Blase ist dauernd gefüllt und der Urin geht durch die Harnröhre tropfenweise ab. Später hält der Junge den Urin bis zu einer halber Stunde und entleert dann ca. $50 \mathrm{ccm}$ im Strahl. I Jahr nach der Entlassung kann der Urin ca. I Stunde gehalten werden, ohne daß es merklich tropft, es werden dann 80-10o ccm entleert. Es ist für den Jungen damals ein Apparat mit einer Pelotte angefertigt worden, durch den auf die Harnröhre an der Peniswurzel ein Druck ausgeübt wird. Dadurch wird der Urin mehrere Stunden zurückgehalten. Weitere Nachrichten von dem Kinde waren leider nicht zu erlangen.

Fall 25. Operateur: Trendelenburg. Bei dem 5jährigen Knaben W. E. besteht eine vollständige Bauch-Blasen-Genitalspalte. Der Abstand der Schambeine beträgt $5 \mathrm{~cm}$.

In der ersten Operation wird die Beckenschaufel ca. $3 \mathrm{~cm}$ von der Synchondrosis durchmeißelt, worauf sich die beiden Schambeine durch seitlichen Druck auf die Beckenschaufel fast ganz zusammenbringen lassen. Es wird versucht, mit dem Wilmsschen Apparat eine Dauerkompression auf die Darmbeinschaufel auszuüben. $\mathrm{Zu}$ diesem Zwecke wird auf beiden Seiten, etwas unterhalb von den Spinae iliacae ant. sup., je eine $5 \mathrm{~cm}$ lange Hautincision angelegt und es werden jederseits drei Stacheln des Apparats mit den dazu gehörigen Holzklötzen auf den Knochen gelegt. Bei dem Zusammenschrauben des Apparats findet aber nur eine geringe Kompression statt, da die Klötze mit den Stiften herunterrutschten. Der Apparat wird wieder abgenommen, sämtliche Wunden werden genäht, die beiden seitlichen außerdem noch drainiert. Das Kind wird im Bett auf die rechte Seite 
gelegt, ein breiter Sack kommt auf die linke Darmbeinschaufel. Die Wunden heilen primär. Das Kind wird bald auf die rechte, bald auf die linke Seite gelagert. Die Belastung wird gut vertragen. Der Abstand der Schambeine verringert sich jedoch nur auf $3,5 \mathrm{~cm}$.

Deshalb wird in einer zweiten Operation auch auf der rechten Seite die Osteotomie ausgeführt, ca. $4 \mathrm{~cm}$ entfernt von der Synchondrosis sacroiliaca. Es entsteht an der Osteotomiestelle ein $11 / 2 \mathrm{~cm}$ breiter Spalt. Die Schambeine lassen sich völlig zusammenbringen. Wieder wird das Kind seitlich gelagert, Belastung der oben befindlichen Beckenschaufel. Die Operationswunde sezerniert zunächst etwas, heilt aber o. B.

In der dritten Operation wird in Beckenhochlagerung die Blase umschnitten und möglichst nach der Tiefe zu mobilisiert. Die Circumcision läuft in zwei parallele, die Sohle der zu bildenden Urethra einschließende Längsschnitte aus, die nach vorn bis zur Glans verlaufen. Die Lösung der Blase von den Schambeinästen ist schwierig. Bei seitlicher Kompression der Beckenschaufel läßt sich der Symphysenspalt ganz zum Verschwinden bringen, es würde aber zu einer starken Quetschung der Urethra und Corpora cavernosa kommen. Es wird deshalb versucht, den Penisstummel in die Höhe zu schlagen und durch eine quere Incision zwischen Skrotum und Peniswurzel von unten her die Schambeine freizulegen. Der Versuch scheitert aber bald an der Schwierigkeit der anatomischen Verhältnisse. Die Naht der Blase und Urethra erfolgt mit einer doppelten Reihe von Lembertschen Katgutnähten. In die Blase wird ein dünnes Drain eingelegt. Durch eine Drahtnaht werden die Schambeinäste so weit zusammengezogen, daß voraussichtlich kein zu großer Druck auf die nach unten verlegte Urethra bzw. den Blasenhals ausgeübt wird. Die Haut wird bis auf die Drainstelle geschlossen. Die Skrotalwunde wird zum größten Teil genäht, zum Teil drainiert.

Diese Operation ist vollkommen erfolglos gewesen. Es geht zunächst der untere Teil, später die ganze Blasennaht auseinander, auch die Naht der Harnröhre hält nicht, so daB derselbe Zustand wie vor der Operation eintritt. In den nächsten Monaten macht das Kind die Masern durch. Nachdem diese abgelaufen sind, wird zunächst der Symphysendraht, der durchgeschnitten ist, entfernt. Dann wird dic Anfrischung und Naht der Spaltränder in derselben Weise wie in der dritten Operation vorgenommen. Diesmal hat die Urethralnaht und Naht der Blase gehalten, bis auf zwei kleine Fisteln am Blasenhals, die durch Lappenbildung gedeckt werden.

Ergebnis: Die Bauch-Blasen-Genitalspalte ist vollständig geheilt. Der Urin kommt spontan aus der Harnröhre in kleinen Portionen; im Strahl, wenn gepreßt wird.

Die letzten Nachrichten stammen vom 5. V. I913. Das Allgemeinbefinden ist ein gutes. Das Kind besucht seit 2 Jahren die 
Schule, trägt ein Urinal, das ihm beim Sitzen etwas unbequem ist. Der Urin fließt dauernd aus einer stecknadelkopfgroßen öffnung, die unterhalb der Harnröhrenmündung liegt, ab. Bei Druck auf die Blase oder beim Pressen wird der Urin in dünnem Strahle entleert. Irgendwelche auf Blasen- oder Nierensteine hindeutende Beschwerden sind nicht vorhanden. Auch röntgenologisch kann kein Steinschatten entdeckt werden. Die von der Operation herrührenden Narben sind vollkommen unempfindlich. Der Penis ist nur mäßig entwickelt. Es besteht ein breiter Symphysenspalt. Der Urin ist etwas trüb und von alkalischer Reaktion, enthält reichlich Zelltrümmer. und zahlreiche weiße Blutkörperchen.

Fall 26. Operateur: Trendelenburg. Bei dem 3jährigen kräftigen Knaben W. A. ist eine vollständige Bauch-Blasen-Genitalspalte vorhanden. Das Kind ist 2 Jahre hindurch mit Gummibindenwicklung um das Becken herum vorbehandelt worden. Dadurch ist der vordem sehr breite Symphysenspalt wesentlich verkleinert worden. Die Blasenschleimhaut liegt noch in Fünfmarkstückgröße vor. Sie ist stark gewulstet und zeigt zahlreiche papilläre Wucherungen, die zum Teil gestielt polypös aufsitzen. Es werden zunächst diese polypösen Wucherungen durch Abquetschen und Thermokauterisieren beseitigt, da sie ein Repositionshindernis bilden.

4 Wochen später wird in Beckenhochlagerung am Übergang in die Haut eine Umschneidung und Auslösung der Wand der Blase zirkulär vorgenommen. Die so gewonnene Hülle ist für die immer noch etwas gewulstete und jetzt offenbar durch Stauung noch mehr spannende hintere Wand zu knapp, so daß noch ein dicker querer Wulst excidicrt werden muB. Dann erst gelingt der VerschluB der Blase. Die Haut wird darüber zusammengezogen. Darauf wird auch dic Anfrischung und Naht der Urethralrinne volizogen. Anfangs heilen Blasen- und Hautnaht, dann geht die letztcre auseinander, während dic Blasennaht zum größten Teil hält und nur im unteren Teile infolge des vielen Schreiens des Kindes auseinander gcsprengt ist. Hier tritt beim Pressen die Blasenschleimhaut zum Teil wieder heraus. Die Epispadienaht ist primär geheilt. Das Kind wird entlassen und die Gummibindenwicklung fortgesetzt.

I Jahr später wird das Kind von neuem aufgenommen. Nur ein kleiner Teil der Blascnschleimhaut und des rechten Ureters liegt frei. Die neugebildete Blase hat also in ihrer früheren Beschaffenheit gehalten. Die vorliegende Schleimhaut ist polypös entartet. Es werden an 12 Stellen Warzen excidiert. 5 Monate später wird nochmals die Anfrischung und Naht der Spaltränder vorgenommen, die wegen der Spannung und Derbheit der Weichteile schwer ist. Die Naht hat nur zum Teil gehalten; beim Pressen tritt der eine Ureter wieder heraus. Das Kind wird vorläufig entlassen; die Bindenwicklung fortgesetzt. 
I Jahr später wird endlich der völlige Verschluß des Defekts durch Anfrischung und Naht der Spaltränder erzielt.

Das Ergebn is ist folgendes: Die Bauch-Blasen-Genitalspalte ist vollkommen geheilt. Das Kind ist völlig inkontinent.

Die letzten Nachrichten stammen aus dem Ende des vorigen Jahres durch briefliche Mitteilung des Vaters. Es ist auch jetzt noch keine Kontinenz eingetreten; das Kind kann wohl auf einige Minuten, wenn es ermahnt wird, den Urin halten, sobald es aber nicht daran denkt, geht der Urin dauernd in Tropfen ab. Das Allgemeinbefinden ist ein sehr gutes, es hat niemals irgendwelche Beschwerden in der Blasen- oder Nierengegend. Die von der Operation herrührenden Narben sind nicht entzündet.

Die Operationsmethode ist auch bei den neueren Fällen im großen und ganzen dieselbe geblieben wie sie Trendelenburg schon zu Anfang anwandte. In der ersten Operation wurden gewöhnlich beide Synchondrosen durchtrennt, was keine Schwierigkeiten machte. Bei einem Erwachsenen wurde einmal die Osteotomie nach Berg vorgenommen, die dadurch schwierig war, $\mathrm{da} \beta$ es an einem geeigneten Instrumente fehlte, welches um die Beckenschaufel herum geführt werden konnte, um so die Weichteile vor Verletzungen zu bewahren. Infolgedessen ist auch in diesem Falle eine Quetschung des Nervus ischiadicus erfolgt, deren Folgen jedoch durch Elektrisieren allmählich wieder beseitigt werden konnten. Auch bei einem Kinde wurde die Osteotomie doppelseitig vorgenommen, sie erwies sich jedoch als viel schwieriger als die Durchtrennung der Synchondrosen.

Nach der Durchmeißelung der Synchondrosen wurde das Kind in den bekannten, von Trendelenburg konstruierten Lagerungsapparat gebettet, durch den eine seitliche Kompression der Beckenhälften ausgeübt wurde. Das Liegen in diesem Apparat wurde im allgemeinen gut vertragen. War dies nicht der Fall, entstanden vielmehr Dekubitalgeschwüre, so wurde das Kind sofort aus dem Apparat herausgenommen und nun seitlich gelagert, und zwar bald auf die rechte, bald auf die linke Seite. Die jedesmal oben befindliche Beckenhälfte wurde dann mit einem Sandsack beschwert und auf diese Weise eine ausreichende seitliche Kompression ausgeübt. In einigen Fällen wurde von vornherein auf die Einbettung in den Apparat verzichtet und gleich die seitliche Lagerung vorgenommen. Der 
Urin wurde jedesmal mit einer Bunsenschen Wasserstrahlluftpumpe abgesaugt, wodurch erzielt wurde, daß das Kind trocken lag und daß die Wunde an der Synchondrose nicht mit Urin in Berührung kam. Die Pumpe wird, wie Trendelenburg selbst in seiner Abhandlung über Heilung der angeborenen Blasenspalte mit Ektopie (Münchn. med. Wochenschr. rgor) angegeben hat, an den Hahn der Wasserleitung angefügt. Durch cinen Gummischlauch steht sie mit einer verschlossenen Glasflasche und durch diese mit einem dünnen Drainrohr in Verbindung, dessen schräg abgestutztes Ende mittelst eines Streifen Heftpflasters in der Inguinalgegend so fixiert wird, daß das Lumen in den kleimen See von Urin eintaucht, welcher sich bei Rückenlage des Kranken in der Gegend des Blasenhalses ansammelt. Unter hörbarem Geräusch wird der Urin in kleinen Quantitäten stoßweise in die Flasche hinein abgesaugt. Unter dem Schutze dieser einfachen Vorrichtung brauchen die Kinder nur selten trocken gelegt zu werden. Die ganz oder fast in ganzer Ausdehnung durch Naht geschlossene Wunde in der Gegend der Synchondrose heilte fast immer per primam. Nur einige Male sezernierte die Wunde, aber nur sehr geringfügig. Die Heilung wurde durch die Infektion kaum verzögert.

Die Einbettung in den Apparat bzw. die seitliche Lagerung wurde so lange fortgesetzt, bis das Becken wieder fest geworden war. Dann wurde in einer zweiten Operation die Anfrischung und Naht der Spaltränder vorgenommen. Wenn irgend möglich, wurde die Naht in einer Etage gemacht. Ließ sich jedoch die Haut nicht über der Spalte zusammenziehen, so mußte darauf verzichtet und die Blasenschleimhaut isoliert genäht werden. In diesen Fällen wurde die Haut nur so weit über der Blase zusammengezogen, als es ohne Spannung möglich war und auf die klaffende Wunde ein Tampon gelegt. Zur Ableitung des Urins wurde entweder in der Mitte oder im oberen Winkel der Blase eine Öffnung gelassen und hier ein Drain eingeführt. Das Nahtmaterial war gewöhnlich Katgut. Es wurden Knopfnähte gelegt, und zwar nach Art der Lembertschen Darmnaht. Sehr viel Sorgfalt wurde immer wieder darauf verwandt, den hinteren Teil der Harnröhre und den Blasenhals möglichst eng zu gestalten. Die Naht der Urethra erfolgte ebenfalls durch Katgutknopfnähte 
nach dem Prinzip der Lembert-Naht. In einigen Fällen war auch nach der Durchtrennung der Synchondrosen die Spannung noch so groß, daß sich die Spaltränder nicht vereinigen ließen. Dann wurden die Schambeine vom Damm aus freigelegt, sowohl absteigender wie horizontaler Schambeinast durchtrennt, die so mobilisierten Knochenstücke einander genähert und durch eine Drahtnaht fixiert. Ganz geschlossen konnte jedoch die Symphyse niemals werden, da man dann hätte befürchten müssen, einen Druck auf den hinteren Teil der Harnröhre und die Corpora cavernosa auszuiben. Durch diese Drahtnaht der Symphyse wurde die Spannung so weit herabgemindert, daß die Naht der Spaltränder jetzt leicht erfolgen konnte.

Primäre Heilung der Blase wurde in keinem der Fälle crziclt. Es mußten vielmehr kleinere oder größere Nachoperationen vorgenommen werden. Diese Fisteln wurden, wenn sie klein waren, durch Anfrischung und direkte Naht verschlossen, waren sie jedoch größer, so wurde Lappendeckung vorgenommen. Auch in den Fällen, in denen eine Zusammenziehung der Haut nicht möglich war, wurde durch eine Lappenplastik die Heilung des Spaltes in den Bauchdecken erzielt.

Die Behandlungsdauer war in den meisten Fällen eine sehr langdauernde. Einesteils durch die Lagerung im Apparat, anderenteils durch Krankheiten (Diphtherie, Masern usw.) kamen die Kinder so stark herunter, daß zwischen den einzelnen Operationen lange Zeit gewartet werden mußte. Ein bleibender Nachteil war bei den Kindern durch die häufigen Operationen und die ofteren Narkosen nicht zu erkennen und hat sich auch, wie die Nachuntersuchung ergeben hat, in den späteren Jahren nicht eingestellt.

Der letzte Fall macht insofern eine Ausnahme, als hier die Naht der Spaltränder erfolgen konnte, ohne daß eine Synchondrosentrennung vorgenommen worden war. Dieses Kind war 2 Jahre lang in der Weise vorbehandelt worden, daß breite Gummibänder um Becken und oberen Teil der Oberschenkel herum gewickelt wurden. Diese Gummibindenwicklung wurde Tag und Nacht fortgesetzt und dadurch erzielt, daß der Abstand der Schambeine wesentlich geringer wurde. Schon in dem Fall 23 war diese gute Wirkung der Bindenwicklung zutage ge- 
treten und der Abstand der Spinae, der vorher $20 \mathrm{~cm}$ betrug, bis auf $13 \mathrm{~cm}$ verringert worden. Die Naht der Spaltränder konnte bei diesem Kinde nicht vorgenommen werden, da es von den Eltern, die allen Vorstellungen unzugänglich waren, nach Hause geholt wurde. Durch diese Bindenwicklung, auf die als erster Passavant aufmerksam gemacht hat, soll ein nicht sehr ergiebiger, aber ständiger Druck auf die noch im Wachsen begriffenen Beckenknochen ausgeübt und dadurch eine Änderung in der Konfiguration der Knochen hervorgerufen werden, in dem Sinne, daß eine allmähliche Annäherung der Schambeine erfolgt, die dann dauernd bestehen bleibt.

Vollständige Kontinenz wurde auch in den neueren Fällen nicht erzielt; bis auf den Fall 24, bei dem eine Kontinenz von einer Stunde erreicht wurde, sind vielmehr alle inkontinent. $\mathrm{Ob}$ bei diesem Kinde die Kontinenz auch in späteren Jahren noch vorhanden gewesen ist, konnte leider nicht ermittelt werden, da der jetzige Aufenthalt sich nicht feststellen ließ. Woran das liegt, daß keine Kontinenz eintritt, läßt sich schwer sagen. Trendelenburg ist uberzeugt, daß auch bei der Blasenspalte ein Sphinkter vorhanden ist. „Der ganze muskuläre und nervöse Apparat ist vollständig und er tritt in Wirkung, sobald die auseinander geklappten Teile zum Ring geschlossen sind und der Ring die normale Enge gewonnen hat.“ So sagt Trendelenburg und führt zum Beweis hierfür verschiedene Gründe an, die recht überzeugend wirken. Zunächst weist er auf einen zur Sektion gekommenen Fall hin, bei dem A. Thierfelder im Bereich der Urethra vor ihrem Eintritt in die Blase und in der Prostata ein reichliches Lager von zirkulär gestellten, glatten Muskelfasern nachweisen konnte. Sodann macht Trendelenburg darauf aufmerksam, daß die durch Naht vereinigte Urethra in den ersten Tagen den Katheter wasserdicht umschließt. Auch wenn das am Katheter befestigte, den Urin ableitende Gummirohr IO-I $5 \mathrm{~cm}$ über dem Niveau der Blase lag, der Urin in der Blase also unter einem gewissen Druck stand, kam kein Tropfen Urin neben dem Katheter heraus, sämtlicher Urin lief durch Katheter und Gummiröhre in die Flasche. Der Verschluß gab erst nach, wenn nach einigen Tagen die Naht nachgab. Diese Tatsache, meint Trendelenburg, spreche für das Vorhanden- 
sein eines unter einem gewissen aktiven Tonus stehenden Muskelringes, wenn auch zugegeben werden muß, daß sie sich auch durch die mechanische Elastizität der Teile erklären läßt. Ferner führt Trendelenburg noch an, daß die operierten Kinder es merken, wenn die Blase stärker gefüllt ist und Drang zum Urinlassen bekommen.

Der Grund für die Inkontinenz ist nach Trendelenburg darin $\mathrm{zu}$ suchen, daß es schwierig ist, eine absolut vollständige prima intentio der Blasennaht zu erzielen. Gerade an der wichtigsten Stelle, wo die Harnröhre in die Blase übergeht, entsteht leicht eine Fistel, und wenn auch der Verschluß an dieser Stelle ohne Fistel erzielt wird, so geht die Vereinigung in der Tiefe doch wieder etwas auseinander. Die Harnröhre und das Orificium externum sind nun zu weit und überdies wird der zu weite Kanal durch den seitlichen Zug der immer wieder etwas nach außen fcdernden Beckenschaufeln in die Quere zu einer Spalte ausgezogen. Der Sphinkterapparat kann dann gar nicht oder nur sehr unvollkommen wirken. Durch Vervollkommnung der Technik müsse sich diese Schwierigkeit beheben lassen.

Als beweisend für seine Ansicht hält Trendelenburg ferner seine Erfahrungen, die er bei Epispadie mit vollständiger Inkontinenz erzielte. Bei dieser Form der MiBbildung, die sich von der Blasenspalte nur graduell unterscheidet, ist ebenfalls immer ein Sphinkterapparat vorhanden und derselbe läßt sich durch ein geeignetes Operationsverfahren zur Funktion bringen. Auch bei der Epispadie mit Inkontinenz findet man, wenn man das Infundibulum untersucht, die Urethra in seinem Bereich abnorm weit. Bei Knaben von 3 Jahren geht der stärkste Katheter spielend in die Blase hinein und in Chloroformnarkose läßt sich sogar der kleine Finger ohne Gewalt in das Infundibulum und das Ostium int. urethrae einschieben. Zieht man den Finger zurück, so fühlt man, wie die gegenüberliegende Blasenwand vorfällt und sich in Gestalt eines förmlichen kleinen Zapfens in das Orificum externum urethrae eindrängt. Spaltet man, am besten in Beckenhochlagerung, die obere Wand des Infundibulum nach der Symphyse zu, so kann man den kleinen Blasenvorfall gut sehen. Daß die zirkulären Muskeln unter diesen Umständen keine Kontinenz bewirken können, ist klar. Auch 
bei einer möglichst energischen Kontraktion der Muskeln wird die zu weite Urethra nur schlaff zusammengefaltet sein und sich bei dem geringsten Druck von der Blase eröffnen. Der sich keilförmig eindrängende Kollaps der Blase wird das Ostium int. urethrae und die Urethra selbst noch mehr und mehr ausdehnen und immer funktionsunfähiger machen. Durch eine operative Verengerung der Urethra im Bereich des Infundibulum muß sich also, meint Trendelenburg, das Vorhandensein des ganzen muskulösen Apparates vorausgesetzt, Kontinenz herstellen lassen. Für die Richtigkeit dieser Anschauung konnte Trendelenburg als beweisend anführen die Erfolge, die er bei der Operation seiner Epispadiefälle hatte.

Auch ich möchte noch zwei weitere von diesem Autor operierte Fälle von Epispadie der Harnröhre mit Inkontinenz bringen, bei denen durch eine Operation Heilung und vollständige Kontinenz erzielt wurde.

Bei dem I 2 jährigen Knaben A. H. bietet der Penis das gewöhnliche Bild der Epispadie. Er ist dabei etwas nach links gerichtet. Die Urethralrinne geht an der Symphyse unter einer queren Hautfalte in das Infundibulum über. Ein mitteldicker Nélatonkatheter läßt sich leicht einführen. Der Urin läuft dauernd ab. Die Symphyse zeigt einen fingerbreiten Spalt, der bindegewebig ausgefüllt ist.

Am 27. V. 1905 wird durch einen in der Mittellinie des Hypogastriums über den Symphysenspalt geführten Schnitt versucht, einen Zugang zu der in der Tiefe eines Trichters liegenden gespaltenen Pars membranacea der Harnröhre zu schaffen. Der Zugang erweist sich als zu eng. Deshalb werden die bindegewebigen Verbindungen durchschnitten und die beiden Beckenschaufeln durch Resektionshaken auseinander gezogen. Jetzt ist die Pars membranacea sehr schön zugänglich. Der vordere Blasenrand wird oberhalb der Symphyse freigelegt und die Blase wird durch eine $\mathrm{I} \mathrm{cm}$ lange Incision eröffnet. Durch diese öffnung wird ein dünnes Drain in die Blase eingeführt. Der Rest der Blasenwunde wird durch zwei Katgutnähte wieder verschlossen. Die gespaltene Urethra wird mit Lembertnähten und der Rest der Wand des Infundibulum mit tiefgreifenden Silberdrahtnähten geschlossen. Nach vorn zu wird zu beiden Seiten der Rinne eine Incision gemacht und der Verschluß der Harnröhre durch tiefe Katgutknopfnähte vorgenommen. Die äußere Haut des Penis bis zur Corona glandis wird mit Silberdraht genäht. Die Wunde heilt per primam bis auf eine winzige Fistel im Blasenhals, die sich aber spontan schließt. Nach 4 Wochen bereits wird der Urin I bis 2 Stunden gehalten. Die Entleerung erfolgt durch die neugebildete 
Harnröhre im Strahl. Einige Wochen später bildet sich eine Fistel an der Corona glandis, durch die aller Urin abläuft. Die Ursache hierfür ist eine Verstopfung der Harnröhrenmündung durch einen kleinen Stein, sobald dieser extrahiert ist, wird der Urin wieder durch die Urethralöffnung entleert. Die Fistel schließt sich von selbst. Bei der Entlassung kann der Urin 2 Stunden gehalten werden.

$\mathrm{N}$ achuntersuchung November 19I3: Der Allgemeinzustand ist ein ausgezeichneter. Schmerzen in der Blasen- und Nierengegend bestehen nicht. Abgang von Steinen ist nicht beobachtet worden. Im Urinieren unterscheidet sich der junge Mann seinen eigenen Angaben nach in keiner Weise von den Kameraden, er braucht nicht öfter Wasser zu lassen. Er hat normales geschlechtliches Empfinden, Erektionen und Ejakulationen; die Immissio penis ist gut möglich. Auch röntgenologisch ist kein Blasen- oder Nierensteinschatten zu entdecken. Der Urin ist vollkommen klar. Der Penis hat fast normale Größe. Die Operationsnarben sind alle reaktionslos.

Hier ist also durch die Operation die Epispadie und die vorher bestehende Inkontinenz vollkommen beseitigt worden.

Auch bei einem zweiten Falle, bei dem $\mathrm{I} 6 \frac{1}{2} \mathrm{j}$ jähr. R. A. ist ein voller Erfolg zu verzeichnen. Bei diesem jungen Manne ist die Epispadie bald nach der Geburt operiert worden. Seitdem kann er den Urin wohl im Liegen und Sitzen halten, aber nicht im Stehen. Er trägt einen Urinal. Da ihm sein Leiden in seinem Berufe hinderlich ist, wünscht er noch einmal operiert zu werden.

Bei dem sonst kräftigen und gesunden Patienten ist der Penis stark verkürzt und etwas nach oben gebogen. Auf seinem Rücken zieht von der Glans bis zwei Querfinger breit oberhalb der Symphyse ein breiter Narbenzug, der besonders in der Symphysengegend stark eingezogen ist. Die Symphyse ist deutlich gespalten. Der Urin fließt durch die Harnröhre ab, er kann im Stehen nicht gehalten werden, wohl aber im Liegen, wenn der Patient wach ist und acht gibt.

Am 14. XII. I906 wird von der Mitte des Dorsum penis bis reichlich fingerbreit oberhalb der Symphyse ein Schnitt gemacht. Die Urethra wird auf dem eingeführten Katheter gespalten. Sie ist sehr weit, $\operatorname{kur}_{z}$ vor dem Blasenhals ist sie ampullenartig erweitert. Bei dem Freilegen des Blasenhalses wird die Blase angeschnitten, die öffnung wird sofort durch einige Nähte verschlossen. Die Harnröhre ist in der Tiefe und an der Symphyse in Narbengewebe fest fixiert und läßt sich schwer loslösen. Die dann gelegten Nähte drohen wegen der starken Spannung durchzuschneiden. Um dies zu vermeiden, werden die Schambeine ganz freigelegt und mit Drahtnähten zusammengezogen. Jetzt gelingt die Naht der Urethra. Die Urethralrinne wird so eng gestaltet, daß ein mitteldicker Katheter eben pas- 
sieren kann. Darüber wird die Haut durch Silberdraht zusammengezogen. Am Blasenhals ist ein kleines Drain in die Blase eingeführt, es wird aber sehr bald fortgelassen, da es sich immer wieder verstopft und nun entleert sich zunächst aller Urin durch die Fistel. Die Urethralwunde heilt primär. Schon nach I4 Tagen fließt der größte Teil des Urins durch die Harnröhre. Der Urin wird alle I-2 Stunden gelassen. Die Fistel verkleinert sich, schließt sich aber nicht ganz, epithelisiert sich vielmehr. Sie wird excidiert und der Defekt durch einen Lappen geschlossen. Gleichzeitig wird auch der Symphysendraht, der durchgeschnitten ist, entfernt.

Ergebnis: Bei der Entlassung kann der Urin im Liegen und Sitzen vollständig gehalten werden, im Stehen träufelt er jedoch noch dauernd $a b$ und wird in einem Urinal aufgefangen.

Später hat sich völlige Kontinenz eingestellt; wie mir seine Mutter Ende November vorigen Jahres mitteilte, dient er augenblicklich sein Jahr als Einjährig-Freiwilliger.

In einem dritten Falle ist der Erfolg nicht so gut. Bei dem 3 jähr. W. G. bietet der Penis wiederum das gewöhnliche Bild der Epispadie. Die Symphyse ist geschlossen. Der Harn träufelt dauernd $a b$, doch sammelt sich im Liegen eine größere Menge an, die dann im Stehen gelassen wird.

Die erste Operation wird im Oktober 1906 gemacht. Zunächst werden Haut und Weichteile bis auf die Symphyse gespalten. Von der Schleimhaut der hinteren Harnröhre und des Blasenhalses wird ein Stück excidiert. Letzterer ist so weit, daß er mit einem dicken männlichen Katheter passiert werden kann. Dann wird die Sectio alta angeschlossen, die leicht über dem eingeführten Metall-Katheter gelingt. Es wird ein Nélatonscher Katheter in die Blase eingeführt und durch einc zirkuläre Naht die Blasenschleimhaut zusammengezogen. Durch Katgutknopfnähte werden an der hinteren Harnröhre und am Blasenhals die Schleimhautränder vereinigt. Dann wird auch der periphere Teil der Urethralrinne angefrischt und mit Katgut genäht.

Io Tage nach der Operation wird in Chloroformnarkose die Blase durch die Blasenfistel gefüllt und es zeigt sich, daß, wenn man die Blasenwunde zuhält, aller Urin durch die neugebildete Harnröhre ab. fließt, wenn der Junge hustet. Sonst wird der Urin gehalten und entleert sich erst wieder durch den eingeführten Katheter. Mit Ausnahme einer kleinen Fistel hinter der Glanspartie ist alles primär geheilt. Im Liegen hält der Junge den Urin gut und entleert ihn dann im Strahl. Im Stehen tropft der Urin dauernd durch die Urethralfistel ab. Mit diesem Befund wird der Knabe entlassen.

Am I3. VI. I9I I wird nochmals der Versuch gemacht, die Inkontinenz zu beseitigen. Die von der früheren Operation herrührenden 
Narben werden excidiert und die Schleimhautränder angefrischt. Dann werden die Symphysenränder freigelegt und der Draht nach Vorbohren hindurch geführt. Vorher waren durch die Blasenwand und das Gewebe im Blaseneingang tiefe Katgutnähte hindurch gelegt, die sich nun, nachdem die Symphyse durch Draht zusammengezogen ist, leicht knüpfen lassen. Darüber wird noch eine zweite Reihe von Katgutnähten gelegt und so der Blasenhals möglichst verengert. Die vordere Harnröhre wird nach Thiersch gebildet. Zum Schluß wird noch eine Blasenfistel angelegt, um den Urin abzuleiten. Die Wunde heilt per primam. Im Liegen wird der Urin gehalten und dann im Strahl bis zu I $50 \mathrm{ccm}$ entleert. Des Nachts über ist der Junge fast immer trocken. Die Blasenfistel hat sich ohne weiteres geschlossen, nachdem der Katheter entfernt worden ist. Auch im Stehen kann eine größere Menge Urin gehalten werden, doch tropft daneben noch immer etwas Urin ab, so daß die Hosen des Jungen immer etwas naß sind. Hinter der Glans bildet sich dann später eine kleine Fistel, durch die sich einige Tropfen entleeren, wenn der Junge den Urin aus der vorderen Harnröhrenöffnung läßt.

Vollständige Kontinenz hat sich auch später nicht eingestellt. Wie mir sein Vater Ende November vorigen Jahres mitteilte, hält der Junge den Urin im Liegen gut, so daß er nachts fast immer trocken bleibt. Den während der Nacht angesammelten Urin kann er, wenn er aufsteht, halten und in ein Nachtgeschirr entleeren. Im Stehen kann er den Urin einige Minuten halten, wenn er dazu ermahnt wird; gewöhnlich tropft dauernd etwas Urin ab. Der Junge trägt eine Bandage mit Leinwandeinlage, kann damit die Schule gut besuchen, macht nach jeder Stunde die Bandage ab und entleert dann eine ziemliche Menge Urin ins Klosett.

Bei einem weiteren Falle ist keine Kontinenz erzielt worden.

Es handelt sich um den $5^{1 / 2}$ jährigen Knaben E. U. Der Penis liegt wie in einer Vertiefung oberhalb der Symphyse, so daß man zunächst nur die Glans mit dem Praeputium auf der Unterseite sieht. Zieht man den Penis am Praeputium hervor, so zeigt es sich, daß er etwa $5 \mathrm{~cm}$ lang ist und auf seiner ganzen Dorsalseite eine Rinne, die mit Schleimhaut ausgekleidet ist, aufweist. Der Urin sickert dauernd hervor. Man sieht am Ende der Urethralrinne die Collic semi, und ganz in der Tiefe eine Hervorwölbung von Schleimhaut, die seitlich zwei Öffnungen aufweist (Trigonum Lieutaudi mit Ureteröffnung). An Stelle der Symphyse ist eine deutliche Einkerbung zu fühlen. Die Röntgenbilder zeigen, daß die Ossa pubis um mehrere Finger breit klaffen. Es handelt sich also hier auch noch um eine partielle Blasenspalte.

Bei der ersten Operation am 7 . V. wird zunächst ein Hautschnitt 
in der Medianlinie angelegt, der in das Infundibulum hinein mündet. Die Bauchwand wird bis auf die Blase gespalten. In den Blasenhals läßt sich leicht ein kleiner Finger einführen. Der Schnitt wird durch den Blasenhals und den untersten Teil der Blase weiter geführt. Senkrecht auf diesen Schnitt zu werden zwei weitere Schnitte gesetzt nach beiden Seiten und damit die medialen Enden der Ossa pubis freigelegt. Dann wird das Kind auf den Bauch gelegt. Die Synchondrosen wer den durch Bogenschnitte freigelegt und mit Messer und Meißel durch. trennt, worauf sich bei seitlichem Druck auf das Becken die Scham. beine leicht zusammenbringen lassen. Nachdem das Kind wieder auf den Rücken gelegt ist, wird der Blasenhals umschnitten und es werden zu beiden Seiten der Schleimhautränder auf dem Penisrücken Incisionen gemacht. Die Naht der hinteren Urethralrinne erfolgt nach dem Prinzip der Lembertschen Darmnaht. Sie wird dadurch so verengt, da $B$ nur ein dünner Katheter passieren kann. Auch der Verschluß des Blasenhalses erfolgt durch Lembertnaht. Um ihn noch mehr zu verengern, ist hier ein Stück Schleimhaut excidiert worden. Bevor am Blasenhals die letzten Fäden geknüpft werden, werden die Schambeine durch Drahtnaht vereinigt. Ein feines Drainrohr wird in die Blase eingelegt und durch die oberen Wundwinkel nach außen geleitet. Die Haut wird mit Silberdraht genäht. Die Heilung erscheint zunächst stark in Frage gestellt, da sich die Wunden mit graugrünlichen Belägen bedecken. Nachdem diese sich abgestoßen haben, zeigt es sich, da $B$ alles gehalten hat bis auf eine Fistel am Blasenhals von der Größe einer Kaffeebohne. Die Wunden über den Synchondrosen sind primär geheilt. Zum Verschließen der Fistel werden mehrere Versuche unternommen, die alle mißlingen. Schließlich gelingt es, sie durch eine Lappenplastik zu decken. Kontinenz wird nicht erzielt.

Die letzten Nachrichten stammen aus dem Jahre 19I3. Der Allgemeinzustand ist ein guter. Der Urin träufelt dauernd durch die Harnröhre $a b$ und wird in einem gut passenden Urinal aufgefangen.

In diesem Falle ist die Blasenwunde nicht primär geheilt. Es hat sich am Blasenhals eine Fistel gebildet. Infolgedessen ist der hintere Teil der Urethra wahrscheinlich wieder auseinander gegangen, so daß der Sphinkter nicht vereinigt ist. Daher ist die Kontinenz nicht eingetreten.

Bei dem vorhergehenden Falle ist die Kontinenz zum größten Teile erreicht worden. Das Kind bleibt die ganze Nacht über trocken, kann auch im Stehen den größten Teil des Urins halten, es gehen nur ab und zu einige Tropfen Urin spontan ab. In den beiden ersten Fällen von Epispadie ist vollständige Kontinenz erzielt worden. In diesen Fällen ist also der vorhandene, 
aber zu weite Muskelring auf das richtige Maß zurückgeführt worden.

Ebenso verhält es sich, meint Trendelenburg, bei dem. hohen Grade der Mißbildung, der vollständigen Harnröhren- und Blasenspalte mit Blasenektopie, nur sind hier die Verhältnisse wegen der Spaltung der Symphyse und der dadurch hervorgerufenen seitlichen Ausspannung der zum Kanal geschlossenen Urethra ungünstiger. Das Ziel, eine ganz normal funktionierende Blase und Urethra auch in diesen schwierigsten Fällen herzustellen, ist auf dem eingeschlagenen Wege aber sicher zu erreichen und wird sicher erreicht werden, wenn die Chirurgie dasselbe unablässig im Auge behält.

Von unseren 7 Fällen sind 2 im Anschluß an die Operation gestorben, bei dem einen, Fall 22, war die Ursache eine Fettembolie, die wohl durch die Durchtrennung der Synchondrosen hervorgerufen sein kann und deshalb der Methode zur Last gelegt werden muß. In dem anderen Falle $(2 \mathrm{I})$ ist das Kind an einer aufsteigenden Pyelonephritis zugrunde gegangen. Auch der bestehende Status lymphaticus dürfte mit zum Tode beigetragen haben. Von den I 8 älteren Fällen sind 5 im Anschluß an die Operation gestorben, Fall I an einer abszedierenden Pyelonephritis, Fall 2 an einer Jodoformintoxikation, die sich, nun wir die Gefahr kennen, leicht vermeiden lassen wird. Fall 8 und I 4 sind an einer Infektion der Synchondrosenwunde zugrunde gegangen. Diese Gefahr dürfte, nachdem wir in der Wasserstrahlpumpe über ein ausgezeichnetes Mittel verfügen, den Urin ständig abzusaugen und dadurch eine Durchtränkung des Verbandes und damit der Wunde mit Urin zu verhüten, auszuschließen, bzw. auf ein Minimum zu reduzieren sein. Der letzte Todesfall ist wohl durch den Operationschok bedingt.

Wie die Nachuntersuchung ergeben hat, ist die Gefahr der späteren Pyelonephritis keine so große; es ist nur einer bisher daran zugrunde gegangen, das ist der Fall 20. Bei den übrigen besteht eine Alkaleszenz des Urins, auch sind im Urin weiße Blutkörperchen reichlicher nachzuweisen als im normalen, jedoch bestehen keine Blasen- oder Nierenschmerzen. Auch Steine können röntgenologisch nicht nachgewiesen werden. Einer von ihnen wurde von mir cystoskopisch untersucht: Die Blase ließ sich leicht 
füllen und faßte über $100 \mathrm{ccm}$. Sehr interessant war, daß die Blase sich fast zu einer Kugel ausdehnte, sie war nur ganz wenig seitlich verzogen. Die vordere Blasenwand war sehr dünn, so daß die Lampe durchleuchtete. Gröbere Veränderungen waren an der Blasenschleimhaut nicht zu sehen. Der Urin wurde in stark wirbelndem Strahl hervorgeschleudert und war vollkommen klar.

Tietze untersuchte einmal cystoskopisch zwei Knaben mit geheilter Blasenspalte und hat diesen Befund in seiner Abhandlung (Zur operativen Behandlung der angeborenen Blasenspalte, Beitrag zur klin. Chirurgie, I8. Bd.) veröffentlicht. Er fand damals bei dem sowohl nach der Trendelenburgschen Methode, als auch bei dem nach der Mikuliczschen Methode operierten Knaben, daß sich die Blase zu einem flachen, blattförmigen Hohlraum umgestaltet hatte, der in dem Trendelenburgschen Falle einen deutlichen, Inkrustationen enthaltenden Recessus bildete. In unserem Falle ließ sich die Blase durch die Füllung fast zu einer Kugel ausdehnen, von Inkrustation war nichts $\mathrm{zu}$ sehen. Damit dürften auch die Schlüsse, die Tietze im Anschluß an den cystoskopischen Befund in bezug auf die nach der Trendelenburgschen Methode operierten Blasenspalten zieht, zum Teil hinfällig sein.

Etwas, worauf Trendelenburg immer wieder hingewiesen hat, war auch bei der Nachuntersuchung der letzten Fälle deutlich zu bemerken, nämlich die gute Form des Penis. Sie ist dadurch bedingt, daß bei der Annäherung der Beckenhälften gegeneinander auch die auseinander gewichenen Crura penis einander genähert werden. Der Penis gewinnt infolgedessen bedeutend an Länge, was therapeutisch insofern sehr wichtig ist, als sich sehr leicht eine Gummiplatte mit Feder am Penis anbringen läßt. Diese Feder drückt auf die hintere Harnröhre und bewirkt, daß der Urin stundenlang zurückgehalten werden kann. Dadurch wird die Sphinkterwirkung geradezu ersetzt. Der Apparat wird, wie die Nachuntersuchung ergeben hat, gewöhnlich gern und ohne Beschwerden getragen. 
IO4 DüNKELOH, Zur Heilung der angeborenen Harnblasen- u. Harnröhrenspalte.

\section{Literaturverzeichnis.}

I. Trendelenburg, Zur operativen Behandlung der Ektopia vesicae. Zentralbl. f. Chir. I895, Nr. 49.

2. - Über Heilung der Harnblasenektopie durch direkte Vereinigung der Spaltränder. Verhandlungen der Deutschen Gesellschaft für Chirurgie I 886, S. I73.

3. - Verhandlungen der Deutschen Gesellschaft für Chirurgie ı887, S. II4.

4. - Zur Heilung der angeborenen Harnblasen- und Harnröhrenspalte. Arch. f. klin. Chir., Bd. 43, S. 3 u. 4.

5. - Uber Heilung der angeborenen Blasenspalte mit Kontinenz des Urins. Münchn. med. Wochenschr. Igor, Nr. 44.

6. - Surgical treatment of epispadias and ectopy of the bladder. Transact of the amer. surg. assoc., t. XXIV, p. I4.

7. - The treatment of ectopia vesicae. Annals of surgery 1906, August.

A usführliche Literaturausgaben:

8. M a z e I, E., Über Blasenektopie und deren operative Behandlung. Beitr. z. klin. Chir., Bd. 23, S. 444 .

9. Katz, Traitement Chirurgical de l'extrophie de la vessie. Paris 1903, G. Steinheil.

Io. D a n e e l, P., Die chirurgische Behandlung der Blasenektopie.

II. Enderlen, Die Blasenspalte. Ergebnisse der Chirurgie und Orthopädie, herausgegeben von Payr und Küttner I9I, 2. 